Bull. Korean Math. Soc. 53 (2016), No. 1, pp. 119-126

http://dx.doi.org/10.4134/BKMS.2016.53.1.119

\title{
SOME OBSERVATIONS ON THE NUMERICAL INDEX AND THE POLYNOMIAL NUMERICAL INDEX
}

\author{
Sun KWANG Kim
}

\begin{abstract}
In this paper, we study both the numerical index and the polynomial numerical index. First, we give a sufficient condition for a Banach space $X$ to have lushness. Second, we study the relation between the renormings of a Banach space and the $k$-order polynomial numerical index. This shows that every real Banach spaces of dimension greater that 1 can be renormed to have 2-order polynomial numerical index $\alpha$ for any $\alpha \in[0,1 / 18)$.
\end{abstract}

\section{Introduction}

The numerical index of a Banach space is a constant relating the norm and the numerical radius of operators on the space. Let us recall the definitions. Given a real or complex Banach space $X, B_{X}$ is the closed unit ball and $S_{X}$ is the unit sphere of $X$. The dual space will be denoted by $X^{*}$, and $L(X)$ is the Banach algebra of all bounded linear operators on $X$.

The numerical radius of an operator $T \in L(X)$ is given by

$$
v(T)=\sup \left\{\left|x^{*}(T x)\right|: x \in S_{X}, x^{*} \in S_{X^{*}}, x^{*}(x)=1\right\} .
$$

We see that $v$ is a seminorm on $L(X)$, and $v(T) \leq\|T\|$ for every $T \in L(X)$. The numerical index of the Banach space $X$ is given by

$$
n(X)=\inf \left\{v(T): T \in S_{L(X)}\right\} .
$$

It is easy to see that $n(X)$ is the greatest constant $k \geq 0$ such that $k\|T\| \leq v(T)$ for every $T \in L(X)$, and so $v$ is a equivalent norm to the operator norm if and only if $n(X)>0$. We can find some related survey and papers in $[7,12,21]$. Later, the numerical index was extended to $k$-homogeneous polynomials, $k$ order polynomial numerical index, in 2006 by Choi et al. [5] as follows

$$
n^{(k)}(X)=\inf \left\{v(P): P \in S_{P\left({ }^{k} X ; X\right)}\right\} .
$$

Received January 2, 2015; Revised May 21, 2015.

2010 Mathematics Subject Classification. Primary 46B04; Secondary 46G25, 46E30, 47A12, 47H60

Key words and phrases. numerical index, polynomial numerical index, polynomial, renorming.

This work was supported by Kyonggi University Research Grant 2014. 
$P\left({ }^{k} X ; X\right)$ denotes the space of $k$-homogeneous polynomials, and numerical radius is defined by

$$
v(P)=\sup \left\{\left|x^{*}(P x)\right|: x \in S_{X}, x^{*} \in S_{X^{*}}, x^{*}(x)=1\right\} .
$$

Recent results on polynomial numerical indices can be found in $[6,13,14,15$, $16,17]$.

Our aim in this paper is to give some observations on these numerical indices.

In Section 2, we study the Banach space with numerical index 1. There were many studies to find a geometric characterization of a Banach space $X$ with numerical index 1 without using operators. Lushness was recently introduced in [4] as a geometrical property of a Banach space which ensures that the space has numerical index 1. Before lush Banach spaces were studied, the basic examples of Banach spaces with numerical index 1 had been known to be almost-CL-spaces $[18,19]$. In fact, lushness is weakest among many isometric properties in the literature which are sufficient conditions for a Banach space to have numerical index 1.

For $x^{*} \in S_{X^{*}}$ and $0<\epsilon<1$, the set of the form

$$
S\left(B_{X}, x^{*}, \epsilon\right)=\left\{x \in B_{X}: \operatorname{Re} x^{*}(x)>1-\epsilon\right\}
$$

is called a slice of the unit ball.

Definition 1.1. A Banach space $X$ is said to be lush if for every $x, y \in S_{X}$ and for every $\epsilon>0$ there is a slice $S=S\left(B_{X}, x^{*}, \epsilon\right) \subset B_{X}, x^{*} \in S_{X^{*}}$, such that $x \in S$ and $\operatorname{dist}(y, \operatorname{aconv}(S))<\epsilon$ where $\operatorname{aconv}(S)=\left\{\sum_{i=1}^{n} \alpha_{i} s_{i}: \sum\left|\alpha_{i}\right| \leq\right.$ $\left.1, s_{i} \in S\right\}$.

On the other hand, a Banach space $X$ is said to have the alternative Daugavet property ( $A D P$ for short) if the norm identity

$$
\max _{|\omega|=1}\|I d+\omega T\|=1+\|T\|
$$

holds for every rank-one operators $T \in L(X)$. Since every Banach space with numerical index 1 has the $A D P$, every lush space has the $A D P$.

We recall that a convex combination of slices of a convex bounded subset $A$ of a Banach space $X$ is a subset of $A$ of the form $\sum_{k=1}^{m} \lambda_{i} S_{i}$ where $\lambda_{i}>0$, $\sum \lambda_{i}=1$ and the $S_{i}$ 's are slices of $A$. For a closed convex bounded subset $A$ of a Banach space $X$, we say that $A$ has small combinations of slices [10] $(S C S$ for short) if every slice of $A$ contains convex combinations of slices of $A$ with arbitrarily small diameter. In this section, we observe that for a Banach space $X$ if $B_{X}$ has $S C S$, then the lushness is equivalent to the $A D P$.

In Section 3, we study the relation between the renormings of a Banach space and the $k$-order polynomial numerical index. C. Finet et al. [8] investigated the values of the numerical index which can be obtained by renorming the space. Their main theorem is that for any Banach space $X$ the set of numerical indices of Banach spaces which are isomorphic to $X$ is an interval. We get the same result for the case of $k$-homogeneous polynomials. More over we conclude that 
for any Banach space $X$ and $k \in \mathbb{N}$ the set of $k$-order polynomial numerical indices of Banach spaces which are isomorphic to $X^{* *}$ is a subset of the set of $k$-order polynomial numerical indices of Banach spaces which are isomorphic to $X$.

\section{SCS and numerical index 1}

It is well known that for a Banach space $X$ with the Radon-Nikodým property the lushness and the $A D P$ are equivalent (see [3, Remark 2.2] and [20, Remark 6]). A. Avilés et al. [1] generalized this fact for a strongly regular Banach space $X$. We recall that a closed convex bounded subset $A$ of a Banach space is said to be strongly regular if every non-empty convex subset $L$ of $A$ contains a convex combination of slices of $L$ of arbitrarily small diameter, and we say that $X$ is strongly regular if $B_{X}$ is strongly regular. Hence, the unit ball of every strongly regular Banach space has $S C S$.

A. Avilés et al. showed the followings. (1) For a Banach space $X$, a separable closed convex bounded subset $A$ of $X$ having $S C S$ is a slicely countably determined ( $S C D$ for short) set. (2) Every Banach space $X$ with the $A D P$ whose unit ball is a $S C D$ set is lush. Hence, we see that separable Banach space with $A D P$ whose unit Ball has $S C S$ is lush. This implies that every strongly regular Banach space with $A D P$ is lush, because lushness and the $A D P$ are separably determined.

The aim of this section is to generalize the last result. Precisely, every Banach space $X$ with the $A D P$ whose unit ball $B_{X}$ has $S C S$ is lush.

Proposition 2.1 ([1, Proposition 4.2]). Let $K\left(X^{*}\right)$ be the weak $k^{*}$ closure in $X^{*}$ of $\operatorname{ext}\left(B_{X^{*}}\right)$, and for every slice $S$ of $B_{X}$ and every $\epsilon>0$, we write

$$
D(S, \epsilon)=\left\{y^{*} \in K\left(X^{*}\right): S \cap \mathbb{T}\left(S\left(B_{X}, y^{*}, \epsilon\right)\right) \neq \phi\right\} .
$$

Then, $X$ has the $A D P$ if and only if for every $\epsilon>0$ and every sequence $\left\{S_{n}\right.$ : $n \in \mathbb{N}\}$ of slices of $B_{X}$, the set $\cup_{n \in \mathbb{N}} D\left(S_{n}, \epsilon\right)$ is weak ${ }^{*}$-dense in $K\left(X^{*}\right)$.

Proposition 2.2 ([10, Corollary III.7]). Let $D$ be a closed convex bounded subset of a Banach space $X$. Suppose $D$ has $S C S$, then for any $x \in D$ and any $\epsilon>0$ there exist positive scalars $\alpha_{1}, \ldots, \alpha_{n}$ with $\sum_{i=1}^{n} \alpha_{i}=1$ and slices $S_{1}, \ldots, S_{n}$ of $D$ such that $\sum_{i=1}^{n} \alpha_{i} S_{i} \subset B(x, \epsilon)$.

Theorem 2.3. Every Banach space $X$ with the ADP whose unit ball $B_{X}$ has SCS is lush.

Proof. Fix any $x, y \in S_{X}$, and $\epsilon>0$. From Proposition 2.2, there exist some positive numbers $\alpha_{i}>0$ and slices $S_{i}$ such that $\sum_{i=1}^{n} \alpha_{i}=1$ and $\sum_{i=1}^{n} \alpha_{i} S_{i} \subset$ $B(y, \epsilon)$. From Proposition 2.1,

$$
A=\left\{y^{*} \in S_{X^{*}}: S_{i} \cap \mathbb{T} S\left(B_{X}, y^{*}, \epsilon\right) \neq \phi, 1 \leq i \leq n\right\}
$$

is a weak*-dense set of $K\left(X^{*}\right)$ and hence $A$ is norming set. Choose $y^{*} \in A$ such that $\operatorname{Re} y^{*}(x)>1-\epsilon$, then for every $1 \leq i \leq n$ there exist $x_{i}$ such that 
$x_{i} \in S_{i} \cap \mathbb{T} S\left(B_{X}, y^{*}, \epsilon\right)$. We can see that $\left\|y-\sum_{i=1}^{n} \alpha_{i} x_{i}\right\|<\epsilon$ which implies $X$ is lush.

Since it is known that a unit ball which is dentable or huskable has $S C S$, we get the following.

Corollary 2.4. Every Banach space $X$ with the $A D P$ whose unit ball $B_{X}$ is dentable or huskable is lush.

\section{Renormings and the polynomial numerical index}

Before we start, let us fix some notations. Given Banach space $X, \mathcal{E}(X)$ denote the set of all equivalent norms on the Banach space $X$, and for $p \in \mathcal{E}(X)$ it will be convenient to denote also by $p$ the dual norm of $p . \mathcal{E}(X)$ is an arcwise connected metric space when endowed with the distance given by

$$
\mu(p, q)=\log (\min \{\alpha \geq 1: p \leq \alpha q, q \leq \alpha p\}) \text { for } p, q \in \mathcal{E}(X) .
$$

We give a norm on $X \times X^{*}$ by $\|(x, f)\|=\|x\|+\|f\|$, and let $\Pi_{p}(X)=$ $\left\{\left(x, x^{*}\right) \in X \times X^{*}: x^{*}(x)=p(x)=p(f)=1\right\} . M(E)$ denote the set of all non-void bounded closed subsets of a metric space $E$ with the distance $d(A, B)=\max \left\{\sup _{x \in A} d(x, B), \sup _{x \in B} d(x, A)\right\}$.

Theorem 3.1 ([2, Theorem 18.3]). The mapping $p \rightarrow \Pi_{p}(X)$ is a continuous mapping of $\mathcal{E}(X)$ in to $M\left(X \times X^{*}\right)$, and is uniformly continuous on each bounded subset of $M\left(X \times X^{*}\right)$.

From this theorem, C. Finet et al. [8] showed that for any Banach space $X$ the set of numerical indices of Banach spaces which are isomorphic to $X$, $\mathcal{N}(X)$, is an interval. Moreover, they conclude that $\mathcal{N}\left(X^{*}\right) \subset \mathcal{N}(X)$ and so $\mathcal{N}\left(X^{* *}\right) \subset \mathcal{N}(X)$. In this section, we get the same result for polynomials.

First, we can easily check that the proof of the above theorem is also valid for any norm $\|(x, f)\|=a\|x\|+b\|f\|$ on $X \times X^{*} a, b>0$, and so we get the following corollary.

Hereafter, $v_{p}(P)$ is the numerical radius of $P$ in the space $(X, p)$, and $n^{(k)}(X, p)$ is the $k$-order polynomial numerical index of $(X, p)$. That is,

$$
\begin{gathered}
v_{p}(P)=\sup \left\{\left|x^{*}(P x)\right|:\left(x, x^{*}\right) \in \Pi_{p}\right\}, \\
n^{(k)}(X, p)=\inf \left\{v_{p}(P): P \in P\left({ }^{k} X ; X\right),\|P\|=1\right\} .
\end{gathered}
$$

And let us define

$$
\mathcal{N}^{(k)}(X)=\left\{n^{(k)}(X, p): p \in \mathcal{E}(X)\right\}
$$

Corollary 3.2. Let $P \in P\left({ }^{k} X ; X\right)$. The mapping $p \in \mathcal{E}(X) \rightarrow v_{p}(P)$ is continuous and uniformly continuous on bounded subsets.

Proof. Let $\epsilon>0$ be given. with $k>1$, define $G_{k}=\{p \in \mathcal{E}(X): \mu(p,\|\cdot\|)<\alpha\}$. Using Theorem 3.1, there exists $\delta>0$ such that

$$
p, q \in G_{k}, d(p, q)<\delta \text { implies } d\left(\Pi_{p}(X), \Pi_{q}(X)\right)<\epsilon .
$$


For any $\eta>0$, we can find $\left(x, x^{*}\right) \in \Pi_{p}(X)$ so that $\left|x^{*} P x\right|>v_{p}(P)-\eta$. Then, $d\left(\left(x, x^{*}\right), \Pi_{p}\right)<\epsilon$ and so there exists $\left(y, y^{*}\right) \in \Pi_{p}$ with $\frac{k^{k}}{(k-1) !}\|x-y\|+\| x^{*}-$ $y^{*} \|<\epsilon$. Then,

$$
\begin{aligned}
& \left|y^{*}(P y)-x^{*}(P x)\right| \\
\leq & \left|y^{*}(P y)-x^{*}(P y)\right|+\left|x^{*}(P y)-x^{*}(P x)\right| \\
\leq & \left\|y^{*}-x^{*}\right\|\|P\|\|y\|^{k}+\left|x^{*} \sum_{j=1}^{k} \check{P}\left(y^{k-j+1}, x^{j-1}\right)-\check{P}\left(y^{k-j}, x^{j}\right)\right| \\
\leq & \left\|y^{*}-x^{*}\right\|\|P\| e^{\alpha k}+k \frac{k^{k}}{k !}\|x-y\|\|P\|\|x\|^{k-j}\|y\|^{j-1} \\
\leq & e^{\alpha k}\|P\| \epsilon .
\end{aligned}
$$

Hence, $v_{p}(P)-\eta<v_{q}(P)+e^{\alpha k}\|P\| \epsilon$. This equation is symmetric and $\eta$ is arbitrary, so $\left|v_{p}(P)-v_{q}(P)\right|<e^{\alpha k}\|P\| \epsilon$.

With the same proof of [8, proposition 2] we can get the following.

Corollary 3.3. Given a Banach space $X$, the mapping $p \rightarrow n^{(k)}(X, p)$ from $\mathcal{E}(X)$ to $\mathbb{R}$ is continuous. Hence, $\mathcal{N}^{(k)}(X)$ is an interval.

Proposition 3.4. Let $X$ be a Banach space of dimension greater than 1 . Then $0 \in \mathcal{N}^{(k)}(X)$ for the real case and $k^{k /(1-k)} \in \mathcal{N}^{(k)}(X)$ for the complex case.

Proof. Take a two-dimensional subspace $Y$ of $X$, and rite $X=Y \bigoplus Z$ for suitable $Z$. For the real Banach spaces, we know that $n^{(k)} \ell_{2}^{2}=0$ for every $k \in \mathbb{N}$ (see [9, Example 2.1]). We can see that $X \simeq \ell_{2}^{2} \bigoplus_{1} Z$ and that $n^{(k)}\left(\ell_{2}^{2} \bigoplus_{1} Z\right)=$ 0 from [5, Proposition 2.8]. For the complex Banach spaces, for each $k$ there exists a 2-dimensional Banach space $Y_{k}$ with $n^{(k)}\left(Y_{k}\right)=k^{k /(1-k)}[11]$. By the same argument in the real case, we can finish our proof.

From the facts that $n^{(k)}(X) \geq k^{k /(1-k)}$ for every complex Banach space and $n^{(k)}\left(X^{* *}\right) \leq n^{(k)}(X)[5$, Theorem 2.3, Corollary 2.15] we get our main result in this section.

Corollary 3.5. For every Banach space $X, \mathcal{N}^{(k)}\left(X^{* *}\right) \subset \mathcal{N}^{(k)}(X)$.

The followings are the spaces whose polynomial numerical indices have been known. In the complex case, $n^{(k)}\left(C_{0}(L)\right)=1$ for every $k \in \mathbb{N}$ and every locally compact space $L$, and $n^{(2)}\left(\ell_{1}\right) \leq 1 / 2$. In the real case, $n^{(2)}\left(c_{0}\right)=n^{(2)}\left(\ell_{1}\right)=$ $n^{(2)}\left(\ell_{\infty}\right)=1 / 2$. Hence, we can see that

$$
\begin{aligned}
& {\left[k^{k /(1-k)}, 1\right]=\mathcal{N}^{(k)}\left(C_{0}(L)\right) \text { for complex case, }} \\
& {[0,1 / 2] \subset \mathcal{N}^{(2)}\left(c_{0}\right), \mathcal{N}^{(2)}\left(\ell_{1}\right), \mathcal{N}^{(2)}\left(\ell_{\infty}\right) \text { for real case. }}
\end{aligned}
$$

Now we recall an isometric condition, called property $\beta$ that was introduced by Lindenstrauss [15]. 
Definition 3.6. A Banach space $X$ is said to have the property $\beta$ with constant $\rho$ if there are two sets $\left\{x_{i}: i \in I\right\} \subset S_{X},\left\{x_{i}^{*}: i \in I\right\} \subset S_{X^{*}}$ and $0 \leq \rho<1$ such that the following conditions hold:

(1) $x_{i}^{*}\left(x_{i}\right)=1, \forall i \in I$.

(2) $\left|x_{i}^{*}\left(x_{j}\right)\right| \leq \rho<1$ if $i, j \in I, i \neq j$.

(3) $\|x\|=\sup _{i \in I}\left\{\left|x_{i}^{*}(x)\right|\right\}$ for all $x \in X$.

The spaces $c_{0}$ and $\ell_{\infty}$ satisfy the above property. In both cases $\rho=0$. Finet et al. [8] calculated the numerical index of a Banach space which has the property $\beta$ with constant $\rho$ to get the fact that $[0,1 / 3[\subset \mathcal{N}(X)$ in the real case and $\left[e^{-1}, 1 / 2[\subset \mathcal{N}(X)\right.$ in the complex case. Similarly, we calculate the 2 -order polynomial numerical index of a Banach space which has the property $\beta$ with constant $\rho$, and find some intervals which contained in $\mathcal{N}^{(2)}(X)$.

Proposition 3.7. Let $X$ be a real Banach space which has the property $\beta$ with constant $\rho$ and write $0<\lambda=(1-\rho) /(1+\rho) \leq 1$. Then

$$
n^{(2)}(X) \geq \frac{\lambda^{2}}{2}
$$

Proof. The proof follows from a modification of the argument in the one of Proposition 5 in [8], but for the sake of completeness we give here its details. Let $\left\{x_{i}: i \in I\right\} \subset S_{X},\left\{x_{i}^{*}: i \in I\right\} \subset S_{X^{*}}$ be the sets which appear in the definition of the property $\beta$ with constant $\rho$. Given $\epsilon>0$ and $P \in P\left({ }^{2} X ; X\right)$, choose $x \in S_{X}$ so that $\|P x\|>\|P\|-\epsilon$. There exist some $j \in I$ such that $\left|x_{j}^{*}(P x)\right|>\|P\|-\epsilon$. Define $t=\left(1+\lambda x_{j}^{*}(x)\right) / 2, z=\lambda x+\left(1-\lambda x_{j}^{*}(x)\right) x_{j}$, and $w=\lambda x-\left(1+\lambda x_{j}^{*}(x)\right) x_{j}$.

We can see easily that $x_{j}^{*}(z)=1, x_{j}^{*}(w)=-1, z-w=2 x_{j}$, and $\|z\|=$ $\|w\|=1$. Since $\lambda x=t z+(1-t) w$, we get

$$
\left|x_{j}^{*}(P \lambda x)\right|=\left|x_{j}^{*}(P(t z+(1-t) w))\right|>\lambda^{2}(\|P\|-\epsilon) .
$$

Hence, from the fact that $2 \check{P}(z, w)=P z+P w-P(z-w)$ we get

$$
\begin{aligned}
\lambda^{2}(\|P\|-\epsilon) & <t^{2}\left|x_{j}^{*}(P z)\right|+(1-t)^{2}\left|x_{j}^{*}(P w)\right|+2 t(1-t)\left|x_{j}^{*} \check{P}(z, w)\right| \\
& \leq t\left|x_{j}^{*}(P z)\right|+(1-t)\left|x_{j}^{*}(P w)\right|+4 t(1-t)\left|x_{j}^{*} P x_{j}\right|,
\end{aligned}
$$

where $\check{P}$ is the associated symmetric bilinear map of $P$. From the facts $t+(1-$ $t)+4 t(1-t) \leq 2$ and $t, 1-t \geq 0$, we can see that one of $\left|x_{j}^{*}(P z)\right|,\left|x_{j}^{*}(P w)\right|$, $\left|x_{j}^{*} P x_{j}\right|$ must bigger than $\frac{\lambda^{2}}{2}(\|P\|-\epsilon)$. This implies that $v(P) \geq \frac{\lambda^{2}}{2}\|P\|$.

For the complex case, we can get improved result.

Proposition 3.8. Let $X$ be a complex Banach space which has the property $\beta$ with constant $\rho$ and write $0<\eta=(1-\rho) \leq 1$. Then

$$
n^{(2)}(X) \geq \frac{\eta^{2}}{2} \text {. }
$$


Proof. Similarly to the case of [8, Proposition 6], we follow the proof of Proposition 3.7. By multiplying by some scalar, we can assume that $x_{j}^{*}(x) \in \mathbb{R}$ and so we can find $a \in[0,1]$ such that $\left|\eta x_{j}^{*}(x)+a i\right|=\left|\eta x_{j}^{*}(x)-a i\right|=1$. We use vectors $z=\eta x+a i x_{j}$ and $w=\eta x-a i x_{j}$, then $z-w=2 a i x_{j}$. The rest of the proof is analogous to the one of Proposition 3.7.

From the fact that every real Banach space can be renormed to have property $\beta$ with constant less than $\frac{1}{2}+\epsilon$ for every $\epsilon>0$, we get the following corollary by using Proposition 3.7.

Corollary 3.9. Let $X$ be a real Banach space of dimension greater than 1 . Then $[0,1 / 18) \subset \mathcal{N}^{(2)}(X)$.

Acknowledgment. The authors wish to express their thanks to the anonymous referee whose careful reading and suggestions have improved the final form of the paper.

\section{References}

[1] A. Avilés, V. Kadets, M. Martín, J. Merí, and V. Shepelska, Slicely countably determined Banach spaces, Trans. Amer. Math. Soc. 362 (2010), no. 9, 4871-4900.

[2] F. F. Bonsall and J. Duncan, Numerical Ranges II, London Math. Soc. Lecture Note Series 10, Cambridge 1973.

[3] K. Boyko, V. Kadets, M. Martín, and J. Merí, Properties of lush spaces and applications to Banach spaces with numerical index 1, Studia Math. 190 (2009), no. 2, 117-133.

[4] K. Boyko, V. Kadets, M. Martín, and D. Werner, Numerical index of Banach spaces and duality, Math. Proc. Cambridge Philos. Soc. 142 (2007), no. 1, 93-102.

[5] Y. S. Choi, D. García, S. G. Kim, and M. Maestre, The polynomial numerical index of a Banach space, Proc. Edinb. Math. Soc. 49 (2006), no. 1, 39-52.

[6] Y. S. Choi, D. García, M. Maestre, and M. Martín, Polynomial numerical index for some complex vector-valued function spaces, Q. J. Math. 59 (2008), no. 4, 455-474.

[7] E. Ed-dari and M. Khamsi, The numerical index of the $L_{p}$ space, Proc. Amer. Math. Soc. 134 (2006), no. 7, 2019-2025.

[8] C. Finet, M. Martín, and R. Payá, Numerical index and renorming, Proc. Amer. Math. Soc. 131 (2003), no. 3, 871-877.

[9] D. García, B. Grecu, M. Maestre, M. Martín, and J. Merí, Two-dimensional Banach spaces with polynomial numerical index zero, Linear Algebra Appl. 430 (2009), no. 8-9, 2488-2500.

[10] N. Ghoussoub, G. Godefroy, B. Maurey, and W. Schachermayer, Some topological and geometrical structures in Banach spaces, Mem. Amer. Math. Soc. 70 (1987), no. 378, iv $+116 \mathrm{pp}$.

[11] L. Harris, The numerical range of holomorphic functions in Banach spaces, Amer. J. Math. 93 (1971), 1005-1019.

[12] V. Kadets, M. Martín, and R. Payá Recent progress and open questions on the numerical index of Banach spaces, Rev. R. Acad. Cienc. Exactas Fís. Nat. Ser. A Mat. 100 (2006), no. $1-2,155-182$

[13] J. Kim and H. J. Lee, Strong peak points and strongly norm attaining points with applications to denseness and polynomial numerical indices, J. Funct. Anal. 257 (2009), no. 4, 931-947.

[14] S. G. Kim, M. Martín, and J. Merí, On the polynomial numerical index of the real spaces $c_{0}, \ell_{1}$ and $\ell_{\infty}$, J. Math. Anal. Appl. 337 (2008), no. 1, 98-106. 
[15] H. J. Lee, Banach spaces with polynomial numerical index 1, Bull. London Math. Soc. 40 (2008), no. 2, 193-198.

[16] H. J. Lee and M. Martín, Polynomial numerical indices of Banach spaces with 1unconditional basis, preprint.

[17] H. J. Lee, M. Martín, and J. Merí, Polynomial numerical indices of Banach spaces with absolute norm, Linear Algebra Appl. 435 (2011), no. 2, 400-408.

[18] A. Lima, Intersection properties of balls and subspaces in Banach spaces, Trans. Amer. Math. Soc. 227 (1977), 1-62.

[19] - Intersection properties of balls in spaces of compact operators, Ann. Inst. Fourier Grenoble 28 (1978), 35-65.

[20] G. López, M. Martín, and R. Payá, Real Banach spaces with numerical index 1, Bull. London Math. Soc. 31 (1999), no. 2, 207-212.

[21] M. Martín, J. Merí, M. Popov, and B. Randrianantoanina, Numerical index of absolute sums of Banach spaces, J. Math. Anal. Appl. 375 (2011), no. 1, 207-222.

Department of Mathematics

KYONGGI UNIVERSITY

SuWON 443-760, Korea

E-mail address: sunkwang@kgu.ac.kr 